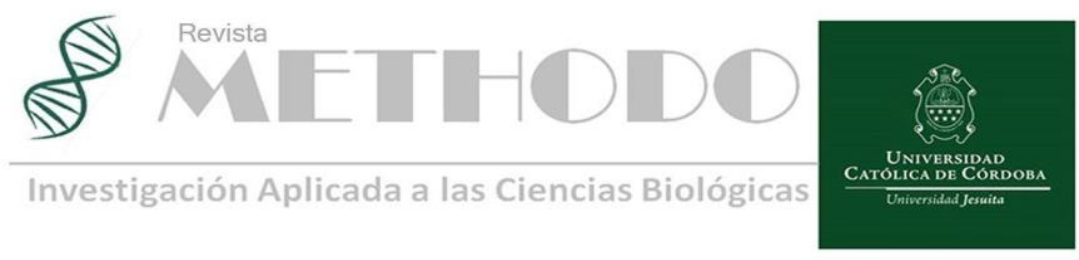

\title{
Abordaje del sobrepeso y la obesidad de niños preescolares en la clínica pediátrica
}

\section{Pediatric approach to overweight and obesity of preschool children}

\author{
María Laura Gonzalvez ${ }^{1,2}$ (iD) Elizabeth Susana De Grandis ${ }^{1}$ \\ 1. Universidad Católica de Córdoba, Facultad de Ciencias de la Salud, Clínica Universitaria Reina Fabiola, Servicio de Pediatría. \\ 2. Hospital de Niños de la Santísima Trinidad de Córdoba, Servicio de Pediatría Ambulatoria. \\ Correspondencia: María Laura Gonzalvez; email: lauragonzalvez@curf.ucc.edu.ar
}

\section{Resumen}

INTRODUCCIÓN: obesidad infantil es un problema de salud pública grave. La prevalencia en Argentina en menores de 5 años para sobrepeso es de $10 \%$ y obesidad del 3,6\%. Se recomienda calcular el Índice de masa corporal (IMC) a todo niño mayor de 2 años durante el control pediátrico. Adquisición desde edades tempranas de hábitos alimentarios y estilo de vida saludables son aspectos insuficientemente considerados en la formación pediátrica.

OBJETIVO: conocer cómo abordan los pediatras el sobrepeso y obesidad en niños preescolares.

MATERIAL Y MÉTODO: Estudio analítico, observacional, transversal. Población médicos especialistas en pediatría que realice atención ambulatoria de niños de 2 a 5 años y trabaja en el Hospital de Niños de la Santísima Trinidad y/o en la Clínica Universitaria Reina Fabiola de Córdoba. Todos los profesionales respondieron un cuestionario para participar.

RESULTADOS: Los resultados se analizaron en base a 155 respuestas. Respecto al cálculo del IMC, el $51 \%$ lo calcula en todos los niños de 2 a 5 años, inclusive. El $76 \%$ de los encuestados respondieron que citan para control del índice de masa corporal. El $42 \%$ refirió que en alguna oportunidad los padres se molestaron frente al planteo del problema de sus hijos. El $17 \%$ refirió haber completado un curso en obesidad y sobrepeso.

CONCLUSIONES: Más de la mitad de los encuestados refiere calcular el IMC en todos los pacientes de 2 a 5 años. Existe asociación entre haber realizado un curso en sobrepeso y obesidad y sentirse capacitado para abordar el tema. Mas de la mitad de los participantes refiere citar para reevaluación del IMC. Las barreras que reconocen son que los padres o pacientes no son receptivos de consejos sobre alimentación saludable y actividad física, factores socioeconómicos y escasez de recursos locales/comunitarios para abordar estos temas.

Palabras claves: obesidad, sobrepeso, padres, pediatras.

\section{Abstract}

INTRODUCTION: childhood obesity is a serious public health problem. Prevalence in Argentina in children under 5 years of age for being overweight is $10 \%$ and obesity is $3.6 \%$. It is recommended to calculate the Body Mass Index (BMI) for all children over 2 years of age during the pediatric control. Acquisition from an early age of healthy eating habits and lifestyles are insufficiently considered aspects in pediatric training. 
OBJECTIVE: know how pediatricians approach overweight and obesity in preschool children.

MATERIAL AND METHODS: Analytical, observational, cross-sectional study. Population is a specialist in pediatrics who provides outpatient care for children from 2 to 5 years old and works at the Hospital de Niños de la Santísima Trinidad and / or at the Reina Fabiola University Clinic in Córdoba. All professionals answered a questionnaire to participate.

RESULTS: The results were analyzed based on 155 responses. Regarding the calculation of BMI, 51\% calculate it in all children from 2 to 5 years old, inclusive. $76 \%$ of those surveyed answered that they cite body mass index control. $42 \%$ said that parents were upset at some point when they raised their children's problem. $17 \%$ reported having completed a course on obesity and overweight.

CONCLUSIONS: More than half of those surveyed reported calculating the BMI in all patients from 2 to 5 years of age. There is an association between having taken a course on overweight and obesity and feeling capable of addressing the issue. More than half of the participants refer to an appointment for a re-evaluation of the BMI. The barriers they recognize are that parents or patients are not receptive to advice on healthy eating and physical activity, socioeconomic factors, and a lack of local / community resources to address these issues.

Keywords: obesity, overweight, parents, pediatricians.

\section{Introducción}

La obesidad infantil es uno de los problemas de salud pública más graves del siglo XXI ${ }^{1}$. A nivel mundial está afectando progresivamente a muchos países de bajos y medianos ingresos, sobre todo en el medio urbano. La prevalencia ha aumentado a un ritmo alarmante. Se calcula que, en 2016, más de 41 millones de niños menores de cinco años en todo el mundo tenían sobrepeso o eran obesos ${ }^{1}$.

La epidemia de obesidad, comienza en etapas tempranas y se arrastra a la adolescencia y adultez con serio impacto en la salud infantil y futura. Junto con la baja talla constituye el problema nutricional antropométrico más prevalente en la población de 0 a 6 años de nuestro país ${ }^{2,3}$.

La prevalencia de obesidad en Argentina en niños y niñas menores de 5 años (0-59 meses) para sobrepeso es de $10 \%$ y la proporción de obesidad en esta población del 3,6\% ${ }^{3}$. Estos indicadores fueron similares para todos los niveles de ingreso ${ }^{3}$. La proporción de niños y niñas de 6 a 60 meses con diagnóstico antropométrico de obesidad y sobrepeso en relación a las curvas de la OMS para la provincia de Córdoba es $6.9 \%$ y $8.5 \%$, respectivamente ${ }^{4}$.

El sobrepeso y la obesidad se definen como una acumulación anormal o excesiva de tejido adiposo. En la práctica clínica el método más utilizado para diagnosticarlos es la antropometría. El índice antropométrico que mejor lo traduce es el índice de masa corporal (IMC) o índice de Quetelet ${ }^{5,6}$. Un IMC entre percentil 85-97 para la edad y sexo es diagnóstico de sobrepeso y por encima del percentil $97 \mathrm{de}$ obesidad.

Se recomienda calcular el IMC a todo niño mayor de 2 años durante el control pediátrico ${ }^{6,7,8}$. Los valores altos del IMC tienen una sensibilidad de moderada a alta del $70 \%$ al $80 \%$ para diagnosticar como verdadera la obesidad y una especificidad del 95\% para diagnosticar como no obeso cuando el paciente no lo es 6,7 .

En la etapa preescolar, y muy influenciado por el ejemplo de los adultos, se comienzan a adquirir preferencias, aversiones y hábitos que serán difíciles de modificar hasta etapas muy posteriores, por lo que el establecimiento de patrones saludables en esta edad es de crucial importancia. Los malos hábitos adquiridos en este periodo pueden tener consecuencias y repercusiones en el medio y largo plazo ${ }^{9}$.

Un meta análisis indica que los padres son propensos a percibir erróneamente la proporción peso/talla de su hijo, especialmente los padres que presentan sobrepeso ${ }^{10,11}$.

Un aspecto poco estudiado en la "epidemia de sobrepeso" es la aceptación por parte de los padres del sobrepeso en las etapas infantiles, ya que frecuentemente en nuestro modelo cultural se confunde sobrepeso con salud. Esta aceptación puede condicionar la ausencia de modificaciones en la actividad física o en los patrones alimentarios que están en la base del sobrepeso del niño ${ }^{12}$. El grado de compromiso familiar es sumamente importante en la efectividad del tratamiento de la obesidad en los niños ${ }^{7}$.

Aunque hay mucha evidencia con respecto a la prevalencia y los efectos de la obesidad, se ha trabajado poco en el abordaje de esta enfermedad por parte de los trabajadores de la salud. Esto es un elemento necesario para combatir la obesidad, ya que la enfermedad requiere un enfoque multidisciplinario, con el conocimiento y la 
experiencia de todos los interesados que juegan un papel importante. La adquisición desde edades tempranas de hábitos alimentarios y un estilo de vida saludables son aspectos insuficientemente considerados en la formación pediátrica general ${ }^{9}$.

La evaluación precisa y adecuada del sobrepeso y la obesidad en niños y adolescentes es un aspecto crítico de la atención médica contemporánea. Sin embargo, los médicos y otros profesionales de la salud pueden encontrar este campo un tanto dificultoso para abordar ${ }^{6}$.

Aunque la Asociación Médica Americana (AMA) clasificó la obesidad como una enfermedad en 2013, se ha encontrado que los médicos continúan tratándolo como un factor de riesgo ${ }^{12,13}$.

El pediatra debe detectar la población de riesgo, sobre la que deberá incidir de manera especial promoviendo la adquisición de hábitos alimentarios saludables y de actividad física. Si el seguimiento es estrecho, podrá detectar precozmente el sobrepeso y la eficacia de las medidas será mayor ${ }^{14}$.

El sobrepeso, la obesidad y las enfermedades conexas son en gran medida prevenibles. Por consiguiente, hay que dar una gran prioridad a la prevención de la obesidad infantil ${ }^{1}$.

El tratamiento de la obesidad en la infancia es la prevención de la obesidad del adulto. Por lo tanto, esta debería ser una de las prioridades de salud pública, con particular énfasis en estimular hábitos saludables de vida en todos los grupos etarios, que incluyen específicamente niños y adolescentes ${ }^{7}$.

La prevención requiere la presencia conjunta de políticas públicas, planeamiento de ciudades y regulación del consumo y la industria de alimentos, entre otros factores. La intervención familiar incluye el aporte de asesoramiento sobre nutrición y actividad física por parte del médico, lo cual mejora la predisposición para educar a los hijos de un modo saludable ${ }^{15}$.

Por todo lo previamente expuesto resulta de importante poder conocer como abordan los pediatras el sobrepeso y la obesidad y la forma en que éstos manejan las recomendaciones sobre nutrición en su consulta diaria en preescolares y conocer qué dificultades identifican en su práctica diaria para el abordaje de estas patologías.

\section{Objetivo}

Conocer cómo abordan los pediatras del Hospital de Niños de la Santísima Trinidad y de la Clínica Universitaria Reina Fabiola de la Ciudad de
Córdoba el sobrepeso y obesidad en niños preescolares.

Objetivos específicos

Valorar si el pediatra refiere detectar el sobrepeso y la obesidad en su práctica diaria (aun en consulta no relacionada) y si notifica a los padres.

Valorar si calculan el Índice de Masa corporal (IMC) en preescolares.

Conocer si los pediatras citan a controles para valorar cambios en el IMC y si los padres cumplen con dicha citación.

Conocer las dificultades que los pediatras identifican en su práctica diaria para el abordaje del sobrepeso y obesidad en preescolares.

\section{Materiales y métodos}

\section{Descripción del ámbito de estudio}

El presente estudio se llevó a cabo entre los médicos pediatras que trabajan en el Hospital de Niños de la Santísima Trinidad y en la Clínica Universitaria Reina Fabiola de la Ciudad de Córdoba, Córdoba, Argentina. Se realizó en el mes de julio de 2020.

Estudio analítico, observacional, transversal.

\section{Selección de técnicas e instrumentos de recolección de datos}

Los datos se recolectaron a través de un cuestionario confeccionado por Butt F, y col. ${ }^{12} \mathrm{y}$ adaptado para la aplicación por médicos pediatras del Servicio de Nutrición Infantil de la Clínica Universitaria Reina Fabiola. El cuestionario consta de 18 preguntas, entre las cuales encontramos 9 preguntas de respuesta binaria, 1 pregunta de escala de Likert de 5 puntos, 6 preguntas semiestructuradas y 2 preguntas de respuesta abierta.

$\mathrm{Se}$ proporcionaron las instrucciones para completar el cuestionario alojado en un formulario de Google vía e-mail y se explicaron los objetivos del presente trabajo.

\section{Plan de análisis de los resultados}

Se describieron las variables cualitativas en porcentaje y se determinó frecuencia. A los fines de asociar las variables se realizaron análisis Chi cuadrado, calculando, cuando resultó pertinente, el Odds Ratio con su correspondiente intervalo de confianza. El almacenamiento y procesamiento de los datos se realizó con el programa estadístico Infostat. En todos los casos el nivel de significación fue del $5 \%$. 
Consideraciones éticas: El estudio se realizó cumpliendo con la normativa de la Declaración de Helsinki y Documento de las Américas de Buenas Prácticas Clínicas (OPS), año 2005, Guía para Investigaciones con Seres Humanos (ANMAT- Res. 1480/11) y Ley Provincial 9694/09. Los autores declaran no tener conflictos de intereses. Se aseguró la protección de datos personales de los profesionales según la Ley 25326/00. El protocolo fue aprobado en el Comité de Investigación Institucional de la Clínica Universitaria Reina Fabiola y en el Comité de Investigación Institucional del Hospital de Niños de la Santísima Trinidad.

\section{Resultados}

Fueron completadas 202 encuestas de las realizadas a los médicos pediatras del Hospital de Niños de la Santísima Trinidad y de la Clínica Universitaria Reina Fabiola durante el mes de julio de 2020.

Se descartaron 46 respuestas por no atender pacientes ambulatorios y 1 respuesta por no atender pacientes de 2 a 5 años, inclusive.

Los resultados se analizaron en base a 155 respuestas de médicos pediatras que realizan atención ambulatoria de niños de 2 a 5 años, inclusive. De las respuestas recibidas el $72 \%$ $(\mathrm{n}=112)$ corresponden al género femenino y el $28 \%(n=43)$ género masculino.

La media de años de práctica profesional, excluyendo los años de residencia, informado por los médicos encuestados fue de 14.06 años, con un rango entre 0 y 43 años.

De los profesionales que respondieron la encuesta el 64\% ( $n=98)$ trabaja en el ámbito público y privado, mientras que el $24 \%(n=38)$ lo hace solo en el ámbito privado y el $12 \%(\mathrm{n}=19)$ solo en el sector público.

Los médicos pediatras que respondieron las encuestas refirieron contar con una subespecialidad pediátrica en el $61 \%(n=95)$ de los casos. En la tabla 1, se describe la distribución de las especialidades referidas.

Respecto al cálculo del IMC, el 51\% ( $\mathrm{n}=79)$ refirió que calcula el IMC en todos los niños de 2 a 5 años, inclusive. El 25\% (n=39) refirió hacerlo solo en niños con aparente sobrepeso u obesidad y el $24 \%(n=37)$ no calcula el IMC en niños de 2 a 5 años, inclusive. Figura 1.
Tabla 1. Distribución por subespecialidades $(\mathrm{n}=95)$

\begin{tabular}{|c|c|c|}
\hline & $\mathrm{n}$ & $\%$ \\
\hline Adolescencia & 1 & 1 \\
\hline Alergia e inmunología & 8 & 8,4 \\
\hline Cardiología & 5 & 5,2 \\
\hline Cirugía & 10 & 10,5 \\
\hline Cuidados paliativos & 1 & 1 \\
\hline Dermatología & 3 & 3,1 \\
\hline Diabetología & 3 & 3,1 \\
\hline Emergentología & 12 & 12,6 \\
\hline Endocrinología & 4 & 4,2 \\
\hline Gastroenterología & 4 & 4,2 \\
\hline Hematología & 1 & 1 \\
\hline Infectología & 4 & 4,2 \\
\hline Medicina interna & 1 & 1 \\
\hline Nefrología & 5 & 5,2 \\
\hline Neonatología & 2 & 2,1 \\
\hline Neumonología & 8 & 8,4 \\
\hline Neurología & 3 & 3,1 \\
\hline No responde & 1 & 1 \\
\hline Nutrición infantil & 2 & 2,1 \\
\hline Oftalmología & 2 & 2,1 \\
\hline Oncología & 1 & 1 \\
\hline $\begin{array}{l}\text { Ortopedia y } \\
\text { traumatología }\end{array}$ & 4 & 4,2 \\
\hline Psiquiatría & 1 & 1 \\
\hline Reumatología & 2 & 2,1 \\
\hline Terapia intensiva & 3 & 3,1 \\
\hline Toxicología & 3 & 3,1 \\
\hline Urología & 1 & 1 \\
\hline
\end{tabular}




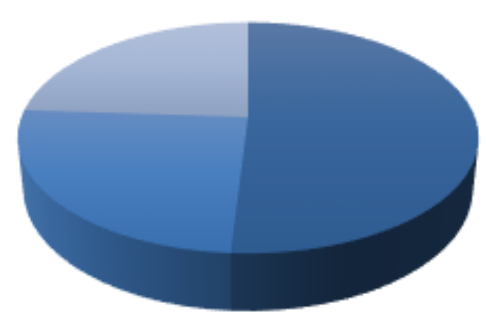

- SI, en todos los pacientes

- SI, solo en pacientes con aparente sobrepeso y obesidad wO calculo enIMC

Figura 1. Calculo del IMC en pacientes de 2 a 5 años, inclusive.

Respecto al cálculo del Índice de masa corporal en niños de 2 a 5 años, inclusive, de los pediatras que realizaron un curso o entrenamiento en sobrepeso y obesidad el $70 \%$ declaró calcular el IMC en todos los pacientes, mientras que aquellos que no realizaron un curso o entrenami- ento el $47 \%$ calcula el IMC en todos los pacientes (OR: 2,69. IC 95\% 1,09-2,69. P 0.04). Los pediatras encuestados con menos de 19 años, inclusive, de experiencia profesional (excluyendo años de residencia) respondieron calcular el IMC a todos los niños de 2 a 5 años en el $48 \%$ de los casos, mientras que aquellos con 20 años o más de experiencia profesional respondieron hacerlo el 58\% (OR 1,49. IC 95\% 0,73-3.03. P 0.35). De los pediatras que cuentan con una subespecialidad, refirieron calcular el IMC en todos los pacientes el $41 \%$, mientras que aquellos que no disponen de una subespecialidad lo hace en el 67\% (OR 0,34. IC 95\% 0,18-0.68. $\mathrm{P}<0.001)$. Tabla 2.

Tabla 2. Factores relacionados al cálculo de IMC $(\mathrm{n}=155)$

\begin{tabular}{|c|c|c|c|c|c|c|c|}
\hline & $\begin{array}{l}\text { Calcula IMC en } \\
\text { todos los pacientes } \\
\text { de } 2 \text { a } 5 \text { años } \\
\text { inclusive }\end{array}$ & & $\begin{array}{l}\text { Calcula IMC solo en } \\
\text { niños de } 2 \text { a } 5 \text { años, } \\
\text { inclusive, con } \\
\text { aparente sobrepeso u } \\
\text { obesidad o No calcula } \\
\text { IMC }\end{array}$ & & & \\
\hline & & $\mathrm{N}$ & $\%$ & $\mathrm{~N}$ & $\%$ & OR (IC) & $\mathrm{P}$ \\
\hline \multirow{2}{*}{$\begin{array}{c}\text { Curso o } \\
\text { entrenamiento } \\
\text { en Obesidad y } \\
\text { sobrepeso }\end{array}$} & $\mathrm{Si}$ & 19 & 70 & 8 & 30 & \multirow[b]{2}{*}{$\begin{array}{c}2,69(1,09- \\
2,69)\end{array}$} & \multirow[b]{2}{*}{0,04} \\
\hline & No & 60 & 47 & 68 & 53 & & \\
\hline \multirow{2}{*}{$\begin{array}{c}\text { Años de } \\
\text { experiencia } \\
\text { profesional } \\
\text { (excluidos años } \\
\text { de residencia) }\end{array}$} & $\begin{array}{c}\text { Meno } \\
\text { s de } \\
20 \\
\text { años }\end{array}$ & 54 & 48 & 58 & 52 & \multirow[b]{2}{*}{$\begin{array}{c}1,49(0,73- \\
3,03)\end{array}$} & \multirow[b]{2}{*}{0,35} \\
\hline & $\begin{array}{c}20 \\
\text { años o } \\
\text { mas }\end{array}$ & 25 & 58 & 18 & 42 & & \\
\hline \multirow[t]{2}{*}{$\begin{array}{c}\text { Subespecialidad } \\
\text { pediátrica }\end{array}$} & $\mathrm{Si}$ & 39 & 41 & 56 & 59 & \multirow{2}{*}{$\begin{array}{c}0,34(0,18- \\
0,68)\end{array}$} & \multirow[b]{2}{*}{$<0,001$} \\
\hline & No & 40 & 67 & 20 & 33 & & \\
\hline
\end{tabular}

El $76 \%(n=118)$ de los médicos pediatras encuestados respondieron que citan nuevamente a los pacientes para control del IMC, mientras que el $24 \%(n=37)$ contestó no hacerlo. El $66 \%$ 
( $\mathrm{n}=103)$ de los médicos consultados refirió que siempre pesquisa el sobrepeso y la obesidad en niños de 2 a 5 años, incluso cuando el motivo de consulta es diferente al tema en cuestión. El 27\% $(n=42)$ refirió hacerlo ocasionalmente y el $5 \%$ $(\mathrm{n}=8)$ y $1 \% \quad(\mathrm{n}=2)$ rara vez y nunca, respectivamente.

Respecto al interés demostrado por los padres frente al problema del sobrepeso y obesidad de sus hijos, el $75 \% \quad(n=116)$ de los médicos encuestados refirió que solo algunos demuestran interés, y frente a la pregunta de si regresan a control tras la citación, el $69 \%(n=107)$ contesto que solo algunos.

El $42 \%(n=65)$ de los médicos encuestados refirió que en alguna oportunidad los padres se molestaron frente al planteo del problema de sobrepeso y obesidad de sus hijos. El 40\% (n= 26) contestó que esta situación le ocurrió entre 3 y 5 veces, el $35 \%(n=23) 1$ a 2 veces y el $25 \%$ $(\mathrm{n}=16)$ en más de 5 oportunidades. El 17\% $(\mathrm{n}=27)$ de los médicos encuestados refirieron haber completado un curso en obesidad y sobrepeso, los cuales corresponden un 66\% $(\mathrm{n}=18)$ a médicos que cuentan con una subespecialidad y a un $34 \%(n=9)$ a pediatras que no cuentan con subespecialidad.

De los médicos que completaron algún curso en sobrepeso y obesidad, el 92\% $(\mathrm{n}=25)$ se considera capacitado para hablar acerca del sobrepeso y obesidad con los padres y el $48 \%$ $(\mathrm{n}=13)$ para realizar un plan de alimentación acorde a la patología mencionada. Mientras que de aquellos que no completaron un curso en sobrepeso y obesidad, el 55\% $\quad(n=70)$ se considera capacitado para hablar acerca del sobrepeso y obesidad con los padres y el $15 \%$ $(n=19)$ para realizar un plan de alimentación acorde a la patología mencionada $(\mathrm{P}=<0.001)$. Tabla 3.

Tabla 3. Relación entre médicos que realizaron un curso en sobrepeso y obesidad y su capacidad acerca del abordaje de la patología con los padres $(n=155)$

\begin{tabular}{|c|c|c|c|}
\hline & $\begin{array}{c}\mathrm{Si} \\
\text { realizo } \\
\text { curso } \\
\mathrm{n}(\%)\end{array}$ & $\begin{array}{c}\text { No realizo } \\
\text { curso } \\
\text { n }(\%)\end{array}$ & $\mathrm{P}$ \\
\hline $\begin{array}{l}\text { Si se considera capacitado para hablar con los padres sobre la } \\
\text { patología } n(\%)\end{array}$ & $25(92)$ & $70(55)$ & \\
\hline $\begin{array}{l}\text { No se considera capacitado para hablar con los padres sobre la } \\
\text { patología n }(\%)\end{array}$ & $2(8)$ & $58(45)$ & $<0,001$ \\
\hline Si se considera capacitado para realizar plan de alimentación n (\%) & $13(48)$ & $19(15)$ & \\
\hline No se considera capacitado para realizar plan de alimentación $\mathrm{n}(\%)$ & $14(52)$ & $109(85)$ & $<0,001$ \\
\hline
\end{tabular}

En la Tabla 4 y Figura 2 se muestran los valores obtenidos de las posibles barreras de los médicos para abordar el sobrepeso y la obesidad en niños de 2 a 5 años, inclusive, durante su práctica profesional.

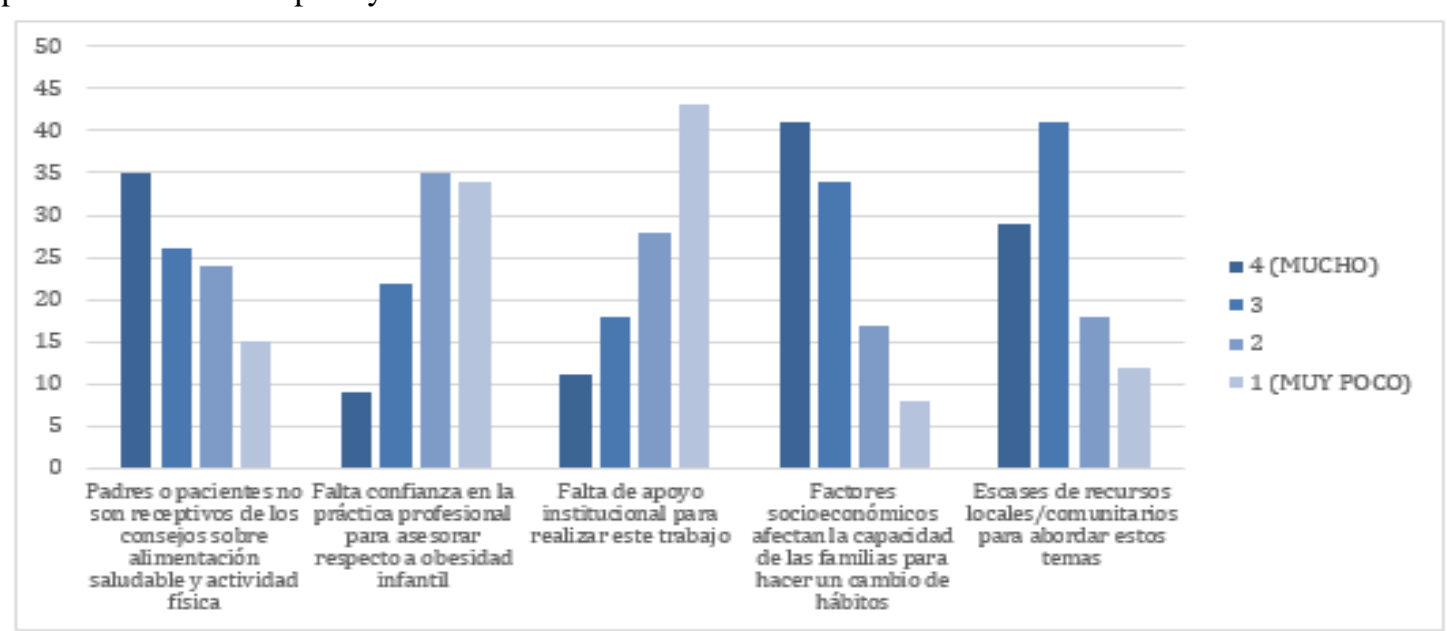

Figura 2. Barreras para abordar el sobrepeso y la obesidad en niños de 2 a 5 años, inclusive, durante la práctica profesional 


\section{Discusión y conclusiones}

La obesidad infantil es uno de los problemas de salud pública más graves del siglo XXI. De acuerdo a los resultados publicados en el resumen ejecutivo de la 2da Encuesta Nacional de Nutrición y Salud (ENNyS) la epidemia de sobrepeso y obesidad es la forma más frecuente de malnutrición y se confirma que continúa aumentando sostenidamente en Argentina ${ }^{3}$. La importancia de conocer cómo abordan los pediatras esta enfermedad, la forma en que éstos manejan las recomendaciones sobre nutrición en preescolares, así como conocer qué dificultades identifican en su práctica diaria puede facilitar el manejo integral de la patología.

De los resultados obtenidos y analizados se desprende que, respecto al cálculo del IMC, lo respondido por los profesionales superó lo informado en otras publicaciones ${ }^{12}$. Este dato podría tener relación con la capacitación en sobrepeso y obesidad, donde los profesionales que respondieron el cuestionario duplicaron los

Tabla 4. Barreras para abordar el sobrepeso y obesidad en niños de 2 a 5 años inclusive durante la práctica profesional $(\mathrm{n}=155)$

\begin{tabular}{|c|c|c|c|c|c|c|c|c|}
\hline & \multicolumn{2}{|c|}{$\begin{array}{c}4 \\
(\mathrm{MUCHO})\end{array}$} & \multicolumn{2}{|c|}{3} & \multicolumn{2}{|c|}{2} & \multicolumn{2}{|c|}{$\begin{array}{c}1 \\
\text { (MUY POCO) }\end{array}$} \\
\hline & $\mathrm{n}$ & $\%$ & $\mathrm{n}$ & $\%$ & $\mathrm{~N}$ & $\%$ & $\mathrm{~N}$ & $\%$ \\
\hline $\begin{array}{c}\text { Padres o pacientes no son receptivos de los } \\
\text { consejos sobre alimentación saludable y } \\
\text { actividad física }\end{array}$ & 55 & 35 & 1 & 26 & 37 & 24 & 22 & 15 \\
\hline $\begin{array}{l}\text { Falta confianza en la práctica profesional } \\
\text { para asesorar respecto a obesidad infantil }\end{array}$ & 14 & 9 & $\begin{array}{l}3 \\
4\end{array}$ & 22 & 55 & 35 & 52 & 34 \\
\hline $\begin{array}{l}\text { Falta de apoyo institucional para realizar } \\
\text { este trabajo }\end{array}$ & 17 & 11 & $\begin{array}{l}2 \\
8\end{array}$ & 18 & 43 & 28 & 67 & 43 \\
\hline $\begin{array}{l}\text { Factores socioeconómicos afectan la } \\
\text { capacidad de las familias para hacer un } \\
\text { cambio de hábitos }\end{array}$ & 63 & 41 & 2 & 34 & 27 & 17 & 13 & 8 \\
\hline $\begin{array}{c}\text { Escasez de recursos locales/comunitarios } \\
\text { para abordar estos temas }\end{array}$ & 45 & 29 & $\begin{array}{l}6 \\
3\end{array}$ & 41 & 29 & 18 & 18 & 12 \\
\hline
\end{tabular}

valores respecto a otras publicaciones frente a la pregunta de si realizaron algún curso o entrenamiento en sobrepeso y obesidad ${ }^{12}$. Existe una asociación significativa entre haber realizado algún entrenamiento y sentirse capacitado para abordar el tema frente a los padres o realizar un plan de alimentación para dicha patología. A pesar de ser esto estadísticamente significativo en ambos casos, tanto sentirse más capacitado para abordar la patología o como armar un plan de alimentación, existe una diferencia de casi el doble entre las dos situaciones, hallándose los médicos menos capacitados para crear un plan de alimentación que para hablar con los padres acerca de la patología. Igual diferencia se mantiene también en aquellos profesionales que no realizaron un curso o entrenamiento en sobrepeso y obesidad. La implicancia de esto radica en la necesidad de aumentar y facilitar la capacitación que se ofrece a los médicos pediatras frente a esta patología para mejorar la confianza profesional y el abordaje integral de los pacientes.

En un artículo recientemente publicado de Silva y cols ${ }^{17}$ el registro del problema de salud en los pacientes pediátricos obesos fue superior comparado con los pacientes con sobrepeso. Se ha descrito que los niños con sobrepeso tienen más riesgo de no ser identificados, por lo que se pierde la posibilidad de intervenir tempranamente en este grupo. Esto es importante al evaluar que, de nuestros profesionales encuestados, hay un tercio $(25 \%)$ que sólo calcula el IMC cuando el paciente tiene aparente sobrepeso y obesidad lo que podría retrasar el diagnóstico y por ende la intervención 
temprana y un $24 \%$ que reconoce no calcular el IMC. Este último porcentaje es también coincidente con otras publicaciones, donde los médicos encuestados refirieron no calcular el IMC o no usar tablas de crecimiento en la consulta en el $28.9 \%{ }^{13}$ o existir un subregistro de la obesidad y sobrepeso en la consulta ${ }^{18}$.

Resulta de gran importancia reconocer entre los resultados que el $66 \%$ de los médicos consultados refirió que siempre pesquisa el sobrepeso y la obesidad en niños de 2 a 5 años, incluso cuando el motivo de consulta es diferente al tema en cuestión. Resulta de gran importancia este resultado ya que podría ser un avance importante respecto a otras publicaciones que describen que se registraron $1.82 \%$ de pacientes con obesidad y sobrepeso frente a un $59 \%$ que realmente cumplían los criterios para el diagnóstico ${ }^{18}$.

Respecto a la revaluación del IMC y la citación para control existe coincidencia en los resultados con respecto a otros estudios donde más de la mitad de los participantes cita para control ${ }^{12}$, y también existe similitud en que, tanto en los resultados obtenidos de nuestros participantes como lo descrito previamente, estos reconocen que mayormente solo algunos de los padres demuestran interés en la patología y regresan al control.

Las principales barreras que reconocen los médicos encuestados son que, los padres o pacientes no son receptivos a los consejos sobre alimentación saludable y actividad física, los factores socioeconómicos afectan la capacidad de las familias para hacer un cambio de hábitos y la escasez de recursos locales/comunitarios para abordar estos temas. Las barreras señaladas por los profesionales en el presente estudio coinciden con otros reportes de la bibliografía ${ }^{12}$ lo cual en parte pone en evidencia que la enfermedad está globalmente distribuida y que enfrenta situaciones similares en varias poblaciones. Los pediatras coinciden en afirmar que las familias con contextos socioeconómicos desfavorables pueden enfrentar mayores dificultades para el cambio de hábitos, mientras que el segundo ENNyS muestra que el sobrepeso y la obesidad fueron similares para todos los niveles de ingreso en niños, niñas y adolescentes ${ }^{3}$. El entorno en el que vivimos actualmente, y que sostiene nuestro estilo de vida, promueve la obesidad y necesita ser cambiado, por lo que se requiere no sólo del esfuerzo de las familias o los equipos de salud, sino de todos los actores de la sociedad ${ }^{19}$.

Múltiples pueden ser las estrategias utilizadas para mejorar el abordaje de esta patología y crear un entorno menos obesogénico, desde optimizar los recursos profesionales, institucionales y comunitarios, intervenciones en escuelas $y$ clubes $^{20}$ hasta modificaciones en el registro de la historia clínica para facilitar el diagnóstico y mejorar la intervención. Los médicos pediatras, como integrantes del equipo de salud, desempeñan un papel fundamental en la prevención y el diagnóstico de la obesidad infantil, por ser ellos quienes entran en contacto con la familia desde edades tempranas y con un acompañamiento usual y prolongado a lo largo de la vida de los pacientes y su entorno. En esto radica la importancia de aumentar y mejorar la formación de los profesionales en este tema, especialmente en el reconocimiento y abordaje temprano de sobrepeso, así como en el manejo integral de obesidad.

\section{Bibliografía}

1. Organización Mundial de la Salud. Estrategia mundial sobre régimen alimentario, actividad física y salud. 2017.Disponible https://www.who.int/es/newsroom/fact-sheets/detail/obesity-andoverweight

2. Enrique O. Abeyá Gilardon, Elvira B. Calvo, Pablo Durán, Elsa N. Longo, Carmen Mazza. Evaluación del estado nutricional de niñas, niños y embarazadas mediante antropometría 1a ed. - Buenos Aires: Ministerio de Salud de la Nación, 2009.

3. Dirección Nacional de Promoción de la Salud y control de Enfermedades no transmisibles. Dirección Nacional de Maternidad, infancia y adolescencia. $2^{\circ}$ Encuesta Nacional de Nutrición y Salud. Principales resultados de los indicadores priorizados. Resumen ejecutivo. Septiembre 2019.

4. Ministerio de Salud. Encuesta Nacional de Nutrición y Salud. Documento de Resultados 2007. Buenos Aires: Ministerio de Salud, 2007.

5. Spear B, Barlow S, Ervin C, Ludwig D, Saelens B, Schetzina K, et al. Recommendations for treatment of child and adolescent overweight and obesity. Pediatrics 2007; 120(Suppl 4): S254-88

6. Nancy F. Krebs, John H. Himes, Dawn Jacobson, Theresa A. Nicklas, Patricia Guilday, et al. Assessment of Child and Adolescent Overweight and Obesity. 
Pediatrics December 2007, 120 (Supplement 4) S193-S228; DOI: https://doi.org/10.1542/peds.20072329D

7. Comité Nacional de Nutrición. Obesidad: guías para su abordaje clínico. Sociedad Argentina de Pediatría. 2015

8. Sociedad Argentina de Pediatría. Programa Nacional de Actualización Pediátrica (PRONAP). Módulo 1. 2017

9. A. Moráis López, V. Martínez Suárez, J. Dalmau Serra, M. ${ }^{a}$ J. Martínez Gómez, L. Peña-Quintana y V. Varea Calderón. Problemas nutricionales percibidos por los pediatras en niños españoles menores de 3 años. Nutr Hosp. 2012;27(6):2028-2047

10. Chávez Caraza, Karla L; Rodríguez de Ita, Julieta; Santos Guzmán, Jesús; Segovia Aguirre, Javán G; Altamirano Montealvo, Diana C; Matías Barrios, Víctor M. Alteración de la percepción del estado nutricional por parte de padres de preescolares: un factor de riesgo para obesidad y sobrepeso. Arch. argent. pediatr; jun. 2016. 114(3): 237242.

11. Rodríguez Martín Amelia, Novalbos Ruiz José P., Villagran Pérez Sergio, Martínez Nieto José M., Lechuga Campoy José L. La percepción del sobrepeso y la obesidad infantil por parte de los progenitores. Rev Esp Salud Pública 2012; 86: 483-494

12. Butt F, Farooq Butt A, Alam F, et al. Perception and Management of Obesity Among Pakistani Doctors. February 28, 2019. Cureus 11(2): e4156. DOI $10.7759 /$ cureus.4156

13. Inge Gies, Bader AlSaleem, Beheshteh Olang, Berkouk Karima, Gamal Samy, et al. Early childhood obesity: a survey of knowledge and practices of physicians from the Middle East and North Africa. BMC Pediatrics (2017) 17:115

14. Lama More RA, Alonso Franch A, GilCampos M, Leis Trabazo R, Martinez Suarez V, Moráis López A et al. Obesidad infantil. Recomendaciones del comité de nutrición de la Asociación Española de Pediatría. Parte I. Prevención. Detección precoz. Papel del pediatra. An Pediatr (Barc) 2006; 65: 607

15. Piazza N, Casavalle P, Kovalskys y colab. Importancia del Tratamiento Adecuado de la Obesidad en Pediatría. Resumen objetivo elaborado por el Comité de Redacción Científica de SIIC en base al artículo Guías de Práctica Clínica para la Prevención, el Diagnóstico y el Tratamiento de la Obesidad. Sociedad Argentina de Pediatría. Archivos Argentinos de Pediatría. May 2011. 109(3):256-266.

16. Stephen R. Daniels. Pediatricians and obesity. The Journal of Pediatrics. August 2019. 211, Pages 1-3

17. Silva C, Mulli V, Caffulli M, Busaniche J. Registro de sobrepeso y obesidad como problema de salud por pediatras de cabecera en una historia clínica electrónica. Arch Argent Pediatr 2020;118(2):132-135.

18. Thomas Katharine, Urrego Fernando. Are Pediatricians Diagnosing Obese Children? Ochsner J. 2017 Spring; 17(1): 80-82.

19. Caballero B. Humans against Obesity: Who Will Win. Disponible en: https://academic.oup.com/advances/arti cle/10/suppl_1/S4/5307222?login=true

20. Brown T, Moore THM, Hooper L, Gao Y, Zayegh A, Ijaz S, Elwenspoek M, Foxen SC, Magee L, O'Malley C, Waters E, Summerbell CD. Interventions for preventing obesity in children. Cochrane Database of Systematic Reviews 2019, Issue 7. Art. No.:

CD001871.DOI: 10.1002/14651858.CD001871.pub4. disponible en: Cochrane Database of Systematic Reviews 2019, Issue 7. Art. No.: $\quad$ CD001871. DOI: 10.1002/14651858.CD001871.pub



